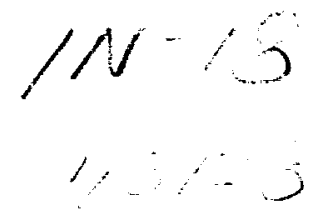

NASA Technical Memorandum 104149

ACTIVE VIBRATION DAMPING OF THE SPACE SHUTTLE REMOTE MANIPULATOR SYSTEM

(NASA-TA-1)4149) ACTIVE UIPKATIUH DAWPING

ง $92-11773$

OF THE SPACE SHUTTLE REMUTL MANIPULATOP

SYSTEM (NASA) I3 P CSCL 220

$p^{2}$

$03 / 180043123$

Michael A. Scott

Michael G. Gllbert

Martha E. Demeo

August 1991

$\hat{A}$

-

N/Sก

National Aeronautics and

Space Administration

Langley Research Center

Hampton, Virginia 23665-5225 



\title{
ACTIVE VIBRATION DAMPING OF THE SPACE SHUTTLE REMOTE MANIPULATOR SYSTEM
}

\author{
Michael A. Scott ${ }^{*}$ \\ Michael G. Gilbert ${ }^{* *}$ \\ NASA Langley Research Center \\ Hampton, VA 23665 \\ Martha E. Demeo ${ }^{\dagger}$ \\ Charles Stark Draper Laboratory \\ Cambridge, MA 02139
}

\begin{abstract}
Controls-Structures Interaction (CSI) is a technology currently under development for application to large flexible space vehicles. The goal of CSI is the improvement of spacecraft performance through active control of the structural dynamic response of the vehicle. This goal is particularly important for modern spacecraft designs where large size and reduced stiffness make structural response a significant contributor to vehicle dynamics. CSI analysis and design methods have been developed to analyze and predict flexible spacecraft performance, but the technology remains largely unvalidated by hardware experiments, demonstrations, or applications, particularly in-space flight applications. One potential application of CSI technology that has been considered is to provide active damping augmentation of the Space Shuttle Remote Manipulator System (RMS). The objective of actively damping the RMS is to demonstrate improved structural dynamic response following payload maneuvers and Shutule reaction control system thruster firings. This paper describes an initial analysis effort to determine the feasibility of controlling the flexible dynamic response of the RMS. The approach to the study is summarized and results from both linear and nonlinear performance analyses of candidate control laws are presented. Results indicate that significant improvement in RMS dynamic response can be achieved through active control if measured RMS tip acceleration was made available for feedback.
\end{abstract}

\footnotetext{
"Spacecraft Controls Branch, Member AIAA

** Spacecraft Dynamics Branch, Senior Member AIAA

$\dagger$ Charles Stark Draper Laboratory, Member AIAA
}

\section{INTRODUCTION}

The Controls-Structures Interaction (CSI) program at the NASA Langley Research Center (LaRC) is dedicated to the development, validation, and application of new technologies for the control of large spacecraft systems which have significant structural flexibility. An important goal of this program is to demonstrate, quantitatively through experiments and applications, the benefits of CSI technology. One potential application which is currently being considered is to actively augment the structural dynamic damping of the Space Shuttle Remote Manipulator System (RMS). The RMS exhibits long periods of oscillatory motion following routine operational maneuvers. This application would provide a direct quantitative measure of the benefit of CSI technology as a part of the CSI program. It could also provide measurable performance improvements in the current RMS, which could ultimatcly have a significant impact on the assembly of Space Station Freedom (SSF).

This paper describes the on-going analysis effort to determine the feasibility of providing active damping augmentation of the RMS following normal payload handling operations. The effort is motivated in part by a study completed by the Charles Stark Draper Laboratory (CSDL) in June 1989. This study examined the use of the Shuttle RMS for a CSI flight experiment. ${ }^{1-2}$ The flight experiment study suggested adding additional sensors to the arm, the installation of a flight experiment computer and hardware in the Shuttle cargo bay, and the use of an instrumented payload at the end of the arm to measure performance. A study of CSI technology benefits for the assembly of Space Station Freedom (assembly scenario prior to the recent SSF restructuring), completed by McDonnell 
Douglas Space Systems Co. in October $1989^{3}$, also motivated the current effort. This study determined that approximately 10 hours of cumulative time would be spent over 15 SSF-assembly Shuttle flights waiting for arm tip motion to damp down to within \pm 1 inch amplitudes following maneuvers with SSF components. The study also showed that a simple two-fold increase in the level of damping of the arm could reduce the cumulative settling time to 4 hours, a reduction in time approximately equal to the programed arm-operation time on a single assembly flight.

The study described in this paper is restricted to consideration of existing RMS hardware if possible, with minimal addition of new hardware only if necessary. The flight experiment computer and the distributed sensors proposed in the CSDL study would be eliminated in favor of using the existing Shuttle General Purpose Computers (GPC's) for control law implementation.

\section{Damping Augmentation Methods}

There are two distinct approaches to reduce residual motions of the RMS following commanded motions. One approach is to reduce the residual oscillations by using input command shaping techniques, as was done by Seering and Singer. ${ }^{4}$ A second approach involves using output feedback of measurements of the system response to derive joint commands designed to damp the residual motions. An example of this approach is the work by Prakash, ${ }^{5}$ Adams, and Appleby, who used a detailed analytical model of the RMS to design model based compensators. Other methods for robust controller design of flexible link arms and nonlinear control methods were suggested by Korolov and Chen ${ }^{6}$ and Kreutz and Jamieson, ${ }^{7}$ respectively.

The advantages of the input shaping approach is that accurate identification of plant parameters, such as frequency and damping, is not critical, and there is no knowledge requirement for the controller influence coefficients. One disadvantage is a significant phase lag between the desired input and corresponding motion of the RMS. This move time penalty is on the order of one period of the first mode of vibration. The operator commands the arm to stop, but the end point will continue to move for a few seconds. This results in the RMS not having the same "feel" as the current RMS when used by a trained operator, and could be detrimental where precise positioning is required. Another disadvantage of command shaping is that it cannot reject unknown disturbances. For example, oscillations of the RMS that result from the Shuttle thruster firings cannot be damped by an input shaping method applied solely to the RMS.
The second approach of employing output feedback through a model based-controller to reduce vibration has been selected for this paper. However, to use a model based-controller, an accurate model of the plant dynamics is required. This model can be obtained either through an extensive analytical model development or through system identification. In this paper, an identified model derived from a nonlinear simulation of the RMS (described below) is used. The advantages of output feedback are that it can reject unknown disturbances regardless of their origin, and the controller can be implemented in such a way as to not change the "feel" of the RMS to trained operators.

\section{Feasibility Study Approach}

The approach to the RMS active damping feasibility study is the following. First, a set of payloads and arm configuration combinations consistent with the types of payloads expected during Space Station Freedom assembly were defined. Second, RMS dynamics and operational characteristics were examined using the nonlinear Draper RMS Simulator (DRS) code. ${ }^{8}$ The code, which is used routinely for predicting arm dynamic motions for on-orbit RMS operations, was obtained from CSDL for this purpose. The simulation code includes models of the RMS structural dynamics, joint servos, motors, gearboxes, and the software modules loaded in the Shuttle GPC for RMS control. The determination of active damping augmentation feasibility involved the design and simulation of candidate damping augmentation control laws. For this purpose, system identification methods were employed on output data from the DRS to identify linear state-space models which closely match the DRS response for specific commanded arm movements. With the linear control design models, various active control law design concepts were evaluated, as were the requirements for feedback sensors to measure arm motions. The final step was the simulation of the active damping control laws in a modified version of the DRS to determine the effects of system nonlinearities and computer time delays.

\section{REMOTE MANIPULATOR SYSTEM}

\section{System Description}

Figure 1 illustrates the elements of the Space Shuttle RMS. ${ }^{9}$ The system is a six-joint telerobotic arm controlled from a panel located on the aft flight deck of the Space Shuttle. These six joints are directly analogous to the joints and freedom of a human arm, defined as shoulder-yaw and pitch, elbow-pitch, and wrist-pitch, yaw, and roll. An end effector for grappling payloads is mounted at the free end of the arm. From the control panel and translational and rotational hand controllers, commands to move the arm are 
processed by the Manipulator Control Interface Unit (MCIU) and the Shuttle GPC to provide electrical signals to drive the joint servo motors. The actual joint servo commands that are generated depend on the selected operational mode, which can be either single-joint mode, one of four manual augmented modes, or several automatic sequence modes. One of the manual augmented modes is normally used for payload operations on-orbit, although the single-joint mode is used for RMS stowing and to avoid joint singularities. Joint angle position and motor shaft rate are measured with an encoder and tachometer, respectively, at each joint, and are returned to the MCIU and GPC for control purposes.

Figure 2 defines the joint movement limits and dimensions of the RMS arm. The arm is shown mounted in the Manipulator Positioning Mechanism (MPM), which is mounted via a swingout joint to the side wall of the Shuttle payload bay. The MPM is used to secure the RMS during launch and reentry of the Shuttle, and is positioned at an angle of $19.4^{\circ}$ relative to the stowed condition during arm on-orbit operations. Also shown is the joint reference coordinate system.

\section{Dynamic Response Studies}

Three study RMS configurations have been adopted for the current study. These configurations are shown in Fig. 3 with the Shuttle PAllet Satellite (SPAS) free-flyer spacecraft as an attached payload. The SPAS payload was used for the dynamic response studies since it is representative of a typical SSF assembly module. These configurations are actual configurations used during the deployment of the SPAS satellite on the STS-07 Shuttle mission. The first of these, referred to as CSI Position 1, is the position of the arm and payload just after release from the cargo bay attachments. CSI Position 2 is the position of the arm and payload after being lifted from Position 1 to a point which completely clears the sides of the cargo bay. CSI Position 3 is the actual deployment positioning at the time of the SPAS release.

The time response data shown in Fig. 4 are typical of the kind of RMS motions encountered during normal arm maneuvers, as predicted by the DRS. The data are the free responses following a 10 -second rotation command to the shoulder-yaw joint in the single joint mode, and the other joints held approximately fixed by the RMS position-hold function. Shown are the lateral displacement of the free end of the arm, the shoulder-yaw joint angle encoder response, and the shoulder-yaw joint rate derived from the motor shaft tachometer. After the command to the RMS is removed, the peak-to-peak free oscillation at the tip of the arm is about 5 inches, while the actual measured joint angle change during the same time is on the order of 0.1 degree. The discrete stepping of the encoder response is due to word length limitations in the Shuttle GPC, indicating that the signal is at the limit of useful resolution. The yaw joint rate is on the order of 3.0 degrees/second, and again has discrete stepping characteristics which is limiting the useful resolution of these data. These types of responses are typical for the study configurations and SPAS payload, and are an indication that the existing RMS sensors may not be completely adequate for active damping augmentation purposes. Because of this, the capability of the DRS to predict three-axis response of an accelerometer package mounted on the SPAS payload was used to simulate an RMS tip mounted accelerometer package. This simulated tip acceleration measurement was used in feedback studies to determine if additional sensor hardware would be beneficial for active damping augmentation of the RMS.

\section{Global Mode Shape Visibility}

Knowledge of the global mode shapes of the RMS was important in assessing the feasibility of active damping augmentation of the RMS. Since mode shapes change with arm geometry, the three CSI configurations were studied. Appraisal was made of mode shapes observability and controllability from the available sensor and actuator suites.

Mode shape information was furnished by CSDL's Eigen DRS. In contrast to the nominal DRS which directly integrates the 25 Degree-Of-Freedom (DOF) of the RMS model, the Eigen DRS transforms the 25 DOF into the frequency regime for evaluation of eigenvalues and eigenvectors. The 25 DOF include a rigid orbiter, RMS joint motion, freeplays at RMS swing out joint and at the grapple point, and torsion and bending in each of the long booms.

Figure 5 shows an exaggerated representation of the second structural mode of the RMS in CSI Position 1 as predicted by Eigen DRS. The predicted frequency of this mode is 0.259 Hertz. This particular mode shape includes a significant amount of upper and lower boom bending. Other modes include significant amounts of joint flexibility and/or orbiter sidewall flexibility, with little boom bending contribution. In order to assess the contributing dynamics of various structural modes, the magnitudes of the eigenvectors of the state equations in the Eigen DRS were plotted. Figure 6 is such a plot, defining the relative contribution of the RMS joints, orbiter sidewall flexibility, and structural deformation to the end point motion. 


\section{CONTROLLER DESIGN AND EVALUATION}

Linear single-input, single-output (SISO), state space models were developed to investigate the damping improvement using local tachometer feedback to the respective joints and tip accelerometer feasibility studies. Multi-input, multi-output (MIMO) state-space models were developed to investigate multivariable state feedback controllers. The methods and results for both cases are presented below.

\section{SISO Studies}

SISO System Identification - Linear SISO state-space models of the RMS were derived from DRS response data using linear system identification methods. The data have been obtained for single joint mode cases with the SPAS payload using the joint rate command as the input, and either the joint tachometer or linear acceleration measurement at the tip of the arm as the output. Assuming a nominal model order of 8 states corresponding to 4 vibration modes, frequency, damping, and influence coefficient parameters were selected to make the model best match the DRS response data in a least-squares sense. In all cases, the system identification process was greatly complicated by the highly nonlinear characteristics of the actual joint hardware. The SISO system identification results for the shoulder-yaw tachometer and the $y$ axis of the simulated tip accelerometer are shown in Figures 7 and 8, respectively. The solid line represents the nonlinear DRS predicted response and the dotted line corresponds to the identified linear model response. These response time histories are the result of a commanded 3-second pulse in shoulder-yaw joint rate. Shoulder-yaw tachometer measurement indicating the effect of this pulse is shown in Fig. 7. These linear models were then used to evaluate the effect on system modes (i.e. damping) from feedback of the tachometer or acceleration measurements through simple gain loop-closures.

SISO Active Damping Results - Figures 9 and 10 show the RMS damping improvement as a function of a scaled gain parameter for feeding back the shoulder-yaw and pitch tachometer measurements, and tip acceleration measurement for CSI position 1. The initial damping values for zero gain for the two joints are different because the joints excite and are able to control different structural modes. For both joints, feedback of the tachometer measurement initially results in a small increase in RMS damping. Feedback of the acceleration measurement in both cases shows larger increases in damping. Also shown in Figure 9 is the result of tachometer feedback as predicted by the nonlinear DRS code, validating the linear model tachometer results. Data shown in Fig. 11 for the shoulderpitch tachometer feedback in CSI Position 3 illustrate the configurational dependence of RMS dynamics. Comparing Fig. 11 with Fig. 9, note the differences in open loop damping and the effect of tachometer feedback for the two configurations. Feedback of tip acceleration is less affected by the configuration change and appears to be more desirable than tachometer feedback for active damping augmentation.

\section{MIMO Studies}

The SISO studies above investigated direct output feedback using tachometer and accelerometer measurements. Multivariable state feedback controllers were also investigated as part of the active damping feasibility study. These controllers were based on identified MIMO linear models of the RMS dynamics. CSDL implemented the MIMO controller logic in the DRS nonlinear simulation so that candidate control laws could be evaluated, including the effects of nonlinear arm dynamics, computer time delays, and existing RMS health and safety software functions.

The MIMO controllers are of the form

$$
\begin{aligned}
& x_{c}(k+1)=A_{c} x_{c}(k)+B_{c} y(k) \\
& u(k)=C_{c} x_{c}(k)+D_{c} y(k)
\end{aligned}
$$

where $A_{C}$ is the compensator dynamics matrix, $B_{C}$ is the control distrubution matrix, $C_{C}$ is the observation matrix, $D_{c}$ is the control feed-through matrix, $x_{c}$ is the state vector and $y$ is the measurement vector consisting of the six joint encoder signals, the six joint motor-rate signals, and three orthogonal RMS tip acceleration signals. The output vector $u$ of the controller consists of six joint rate commands.

MIMO System Identification - The technique used for MIMO system identification was the Observer/Kalman Filter Identification Method (OKID), 10,11 which has recently been developed at the NASA Langley Research Center. (The OKID method, in addition to identifying the state-space matrices A, B, C, and D, also identifies an observer gain matrix $M$ for the system. This identified observer is used later for control law design). Three models were derived, corresponding to the three study positions of the RMS. All three models had three inputs corresponding to the shoulder-yaw, shoulder-pitch, and elbow-pitch rate commands, and three outputs corresponding to the three-axis acceleration at the tip of the RMS. The three models are sixth order, corresponding to three structural modes. Prior to the system identification, the DRS simulation acceleration data were processed through a first-order lowpass filter with a break frequency of $0.2 \mathrm{~Hz}$. 
To perform the identification of the MMO models using the OKID method, data from several DRS simulation runs were aggregated (stacked). Each DRS run involved the simulation of output response for the desired configuration to one of the three input joints, either shoulder-yaw or pitch, or elbowpitch. In each run, the selected joint was given a 3-second pulse rate command, which was intended to excite the low frequency modes of the RMS. The input command and response data were then stacked to allow the OKID algorithm to identify a single model representing the response of the RMS to any of the three inputs.

Results of the MIMO system identification are shown in Figures 12 and 13. Shown are comparisons of the nonlinear DRS simulation response data with one of the MIMO identified models. Figure 12 shows the arm tip position following the 3-second pulse shoulder-yaw rate command (from 0 to 3 seconds in the plot) for CSI position 1. In this figure both the DRS nonlinear simulator (solid line) and the identified linear model (dashed line) match so closely that the curves overlap. Figure 13 illustrates the $y$ axis of the tip acceleration for both the DRS nonlinear simulator (solid line) and the identified linear model (dashed line) for the same 3-second pulse command. A summary of the identified frequency and damping of the three structural modes for the three study configurations are given in Table 1 .

Mimo Controller Design - The multivariable vibration suppression control law for each configuration was developed using the frequency weighted Linear Quadratic Regulator (LQR) design method of Gupta. ${ }^{12}$ Prior to the frequency weighted $L Q R$ regulator design, a digital high-pass filter was prepended to the MIMO identified model to reject steady-state bias as would be encountered in feeding back accelerometer measurements in a real system. This filter had the digital form

$$
N(z)=\frac{\tau_{1} z+\tau_{2}}{\tau_{3} z+\tau_{4}}
$$

where the constants $\tau_{1}$ through $\tau_{4}$ have the values 0.9707 , $-0.9707,1$, and -0.9414 respectively. The vlaues for this filter correspond to a first order high pass filter with a break frequency of $.12 \mathrm{~Hz}$. The high pass filter in series with the MIMO identified model are transformed to one discrete statespace model with corresponding $\hat{A}, \hat{B}, \hat{C}, \hat{D}$ state-space matrices.

For control purposes, a fixed gain regulator of the form

$$
u(k)=-G \hat{x}(k)
$$

was used, where $u$ is the vector of joint rate command signals. The state estimate $\hat{x}$ was obtained from an observer of the form

$$
\hat{x}(k+1)=\hat{A} \hat{x}(k)+\hat{B} u(k)+M[y(k)-\hat{C} \hat{x}(k)]
$$

where $y$ is the tip accelerometer measurement and the observer gain $M$ was identified from the OKID system identification method.

To obtain the optimal gain $G$, the model with the prepended filter was used in a frequency weighted $L Q R$ design with a weighted cost function of the form

$$
J=\sum_{k=0}^{N} y^{T} Q y+u^{T} R u
$$

where $Q$ is the output weight matrix, and $R$ is the control weighting matrix. The numerical values of $Q$ and $R$ were determined using an iterative design procedure on the linear model which avoided actuator saturation. The final values used in the design are $Q=\operatorname{diag}\left\{\begin{array}{lll}0.002 & 0.002 & 0.002\end{array}\right\}$ and $R=\operatorname{diag}\left\{\begin{array}{lll}0.01 & 0.01 & 0.05\end{array}\right\}$. Using

$$
y=\hat{C} \hat{x}+\hat{D} u
$$

the performance index (5) was recast:

$$
\begin{aligned}
J= & \sum_{k=0}^{N} \hat{x}^{T} \hat{C}^{T} Q \hat{C} \hat{x}+2 \hat{x}^{T} \hat{C}^{T} Q \hat{D} u \\
& +u^{T}\left(\hat{D}^{T} Q \hat{D}+R\right) u
\end{aligned}
$$

The optimal feedback gain $G$ which minimizes the performance index $\mathrm{J}$ in equation (7) was found using Matrix $_{\mathbf{X}}{ }^{13}$ software tools.

MIMO Active Damping Results - The multivariable LQR controller with observer was evaluated on the DRS nonlinear simulator. The tip position following a 3-second shoulder-yaw pulse rate command is shown in Fig. 14. In addition, after 90 seconds, Shuttle thruster firing was simulated for 6 seconds. The solid line represents standard RMS operation, the dotted line represents actively damped performance. The time required to damp the tip oscillation to \pm 1 inch is decreased by a factor of 3 . Note that the steady state value of the tip displacement is the same as the nominal value. This is accomplished without using position feedback by the use of the high pass filter appended to the observer plant model in the regulator design. The shoulder-yaw servo torque following the 3-second shoulder- 
yaw pulse rate command is shown in Fig. 15. The solid line represents standard DRS operation, the dotted line represents closed loop performance. In this time history, the controller has the effect of reducing the applied torque by a factor of 2 . This provides an added potential benefit of also reducing the structural stress in the arm following routine maneuvers involving either joint commands or Shuttle thruster firings.

\section{CONTROLLER IMPLEMENTATION IN RMS SOFTWARE}

Based on the recommendations of CSDL, a potential means of implementing an active damping augmentation controller in the Shuttle GPC software was identified. This strategy, illustrated in Fig. 16, allows use of all existing RMS health and safety monitoring functions, in an effort to simplify flight development work. The Control Structure Interaction Controller (CSIC), as it is called, would be a software module which acts as a preprocessor to the existing RMS Command Output Processor (COP). It would be turned on and off by the executive function of the existing software by a flag which would activate the CSIC when RMS joint move commands are zeroed. Using motor rate and/or acceleration feedback measurements, the CSIC would damp the free response of the arm to some level, at which time the normal position-hold function of the arm would be activated. With this implementation, the active damping function of the controller could be expanded to damp RMS motions following Shuttle thruster firings as well.

\section{CONCLUDING REMARKS}

An analytical study to determine the feasibility of actively augmenting the damping of the Shuttle RMS has been summarized. SISO and MIMO linear models were identified and used to design direct output feedback and multivariable controllers. The controller and logic were implemented in the DRS nonlinear simulation, where candidate control laws were evaluated including the effects of nonlinear arm dynamics, computer time delays, and existing RMS health and safety software functions. Based on initial results, active damping of the RMS appears feasible using the existing joint actuators and Shutle computers and software. However, some additional feedback sensors in the form of accelerometers located at the tip of the arm are required.

The controller developed for this system does not change or delay the trained operator input command to move the arm, thus, the "feel" of the arm is not altered. In addition, the controller incorporates output compensation to ensure that the robotic manipulator is in the same final position as when the vibration suppression strategy was initiated. This is accomplished with three tip accelerometers, and not with any endpoint position measurements. The MIMO control system, when evaluated on the nonlinear DRS, demonstrated significant improvement over the present arm performance: (1) Damping level is improved by a factor of 3; (2) Peak joint torque is reduced by a factor of 2 following Shuttle thruster firings. Future evaluation of this controller is planned on the Shutule Engineering Simulator (SES) at the Johnson Space Center. Based on the results of the SES simulations, the RMS community (operators and users) will decide whether or not it is desirable to advocate a flight demonstration.

\section{REFERENCES}

${ }^{1}$ Demeo, M. E., "Remote Manipulator System (RMS) Based Controls - Structures Interaction (CSI) Flight Experiment Feasibility Study." NASA CR 181952 , January 1990.

${ }^{2}$ Demeo, M. E., Fontana, A., and Bains, E. M., "Shuttle Remote Manipulator System (RMS) - Based Controls Structures Interaction Flight Experiment." Fourth NASA / DOD Conference on Controls - Structures Interaction Technology, Orlando, Florida, November 5-7, 1990.

${ }^{3}$ Lamb, B. A., and Nowlan, D. R., "Benefits of Control Structure Interaction (CSI) Technology to Space Station Assembly by Flexible Robotic Systems (RS)." CRAD NAS1-18763, November 1989.

${ }^{4}$ Singer, N.C., and Seering, W.P., "Preshaping Command Inputs to Reduce System Vibration" Journal of Dynamic Systems, Measurement, and Control, Vol. 112, March 1990, pp. 76-82.

${ }^{5}$ Prakash, O., Adams, N.J., and Appleby, B.D., "Multivariable Control of Space Shuttle Remote Manipulator System," Proceedings of the AIAA Guidance, Navigation and Control Conference, New Orleans, Louisiana, Aug.1991.

${ }^{6}$ Korolov, V.V., and Chen, Y.H., "Controller Design Robust to Frequency Variation in a One Link Flexible Robot Arm," Journal of Dynamic Systems, Measurement. and Control, Vol. 111, 1989, 9-14.

${ }^{7}$ Kreutz, K., and Jamieson, R.S., "Linearization of Robot Manipulators," NASA Tech Brief, vol. 11, no. 8, item \#114, 1987.

${ }^{8}$ Gray, C., et al., "Validation of the Draper RMS Simulation (DRS) Against Flight Data." CSDL-R-1755, Volumes 1-2, April 1985.

${ }^{9}$ NSTS 07700, Volume XIV, Appendix 8, System Description and Design Data - Payload Deployment and Retrieval System, Revision J, May 1988.

${ }^{10}$ Phan, M., Horta, L.G., Juang, J.-N., and Longman, R.W., "Linear System Identification Via an Asymptotically Stable Observer," Proceedings of the AIAA Guidance, Navigation and Control Conference, New Orleans, Louisiana, Aug. 1991. 
${ }^{11}$ Juang, J.-N., Horta, L.G., Phan, M., and Longman, R.W., "Identification of Observer and Kalman Filter Markov Parameters: theory and Experiments," Proceedings of the AIAA Guidance, Navigation and Control Conference, New Orleans, Louisiana, Aug.1991.
${ }^{12}$ Gupta, N.K., "Frequency-Shaped Cost Functionals: Extensions of Linear-Quadratic Gaussian Design Methods," Journal of Guidance and Control, Vol. 3, Nov.-Dec. 1980, pp. 529-535.

${ }^{13}$ MATRIX $_{\mathrm{x}}$ Control Design Module, Edition 7 - January 1990. Integrated Systems Inc.

Table 1 - Frequency and damping of identified modes

CSI Position 1

CSI Position 2

CSI Position 3

\begin{tabular}{|c|c|c|c|c|c|c|}
\hline Mode & Freq. (Hz.) & Damping & Freq. (Hz.) & Damping & Freq. (Hz.) & Damping \\
\hline 1 & 0.180 & 0.118 & 0.170 & 0.086 & 0.138 & 0.129 \\
2 & 0.199 & 0.113 & 0.214 & 0.202 & 0.198 & 0.379 \\
3 & 0.488 & 0.421 & 0.352 & 0.593 & 0.363 & 0.755 \\
\hline
\end{tabular}

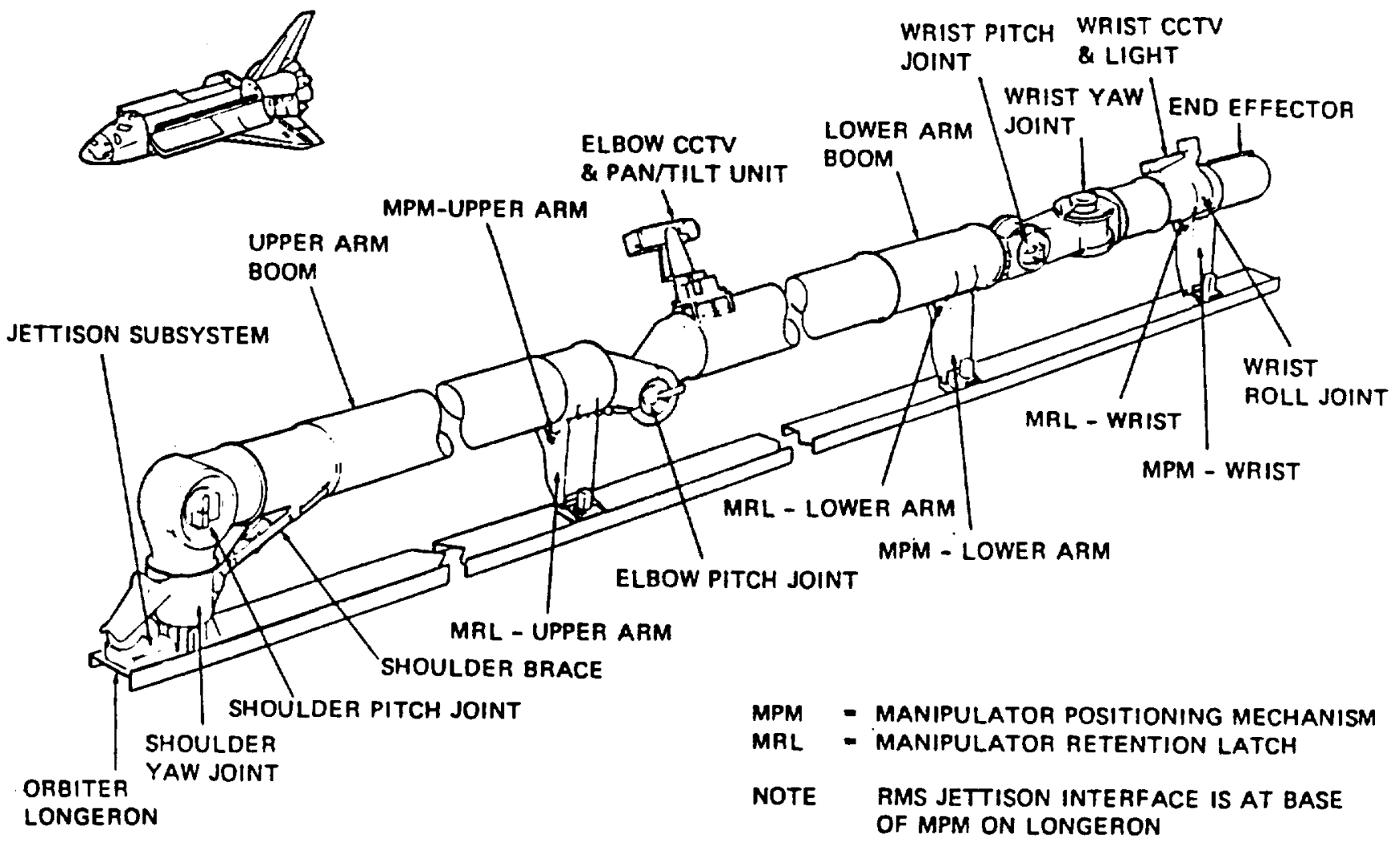

Figure 1 - Space Shuttle Remote Manipulator System (RMS). 


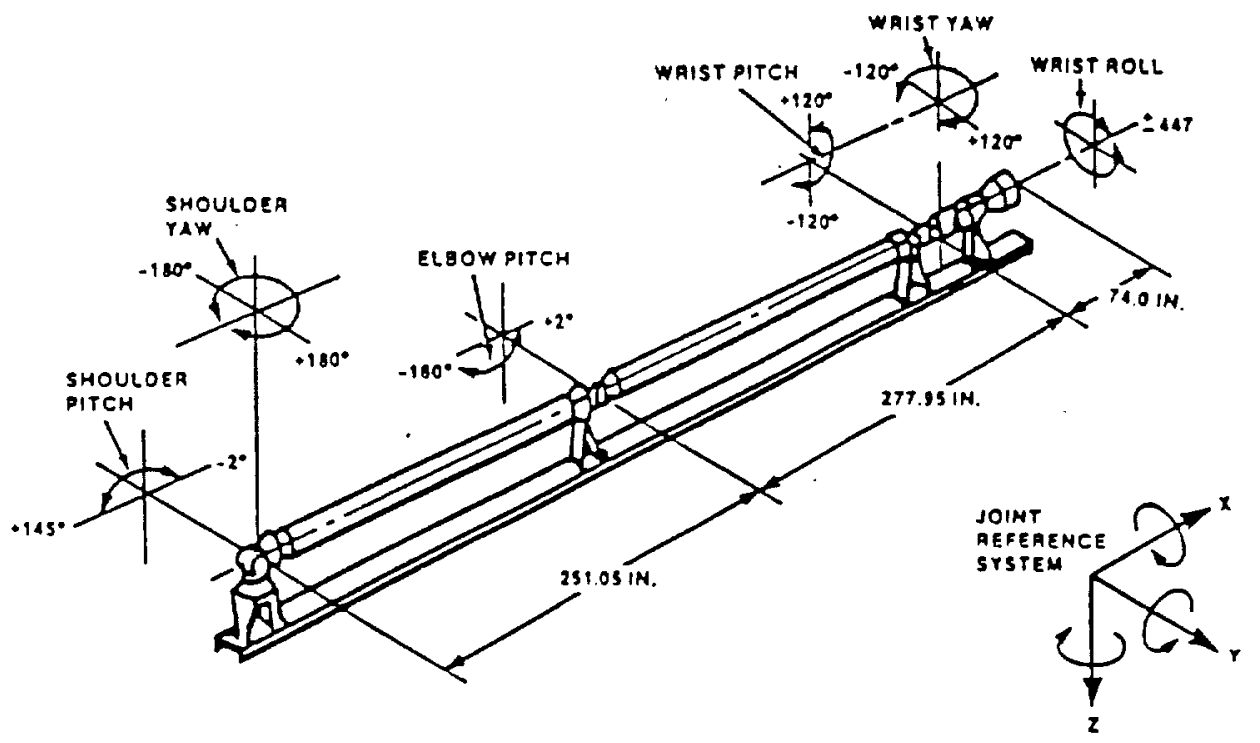

Figure 2 - RMS Dimensions, joint limits, and Coordinate System.
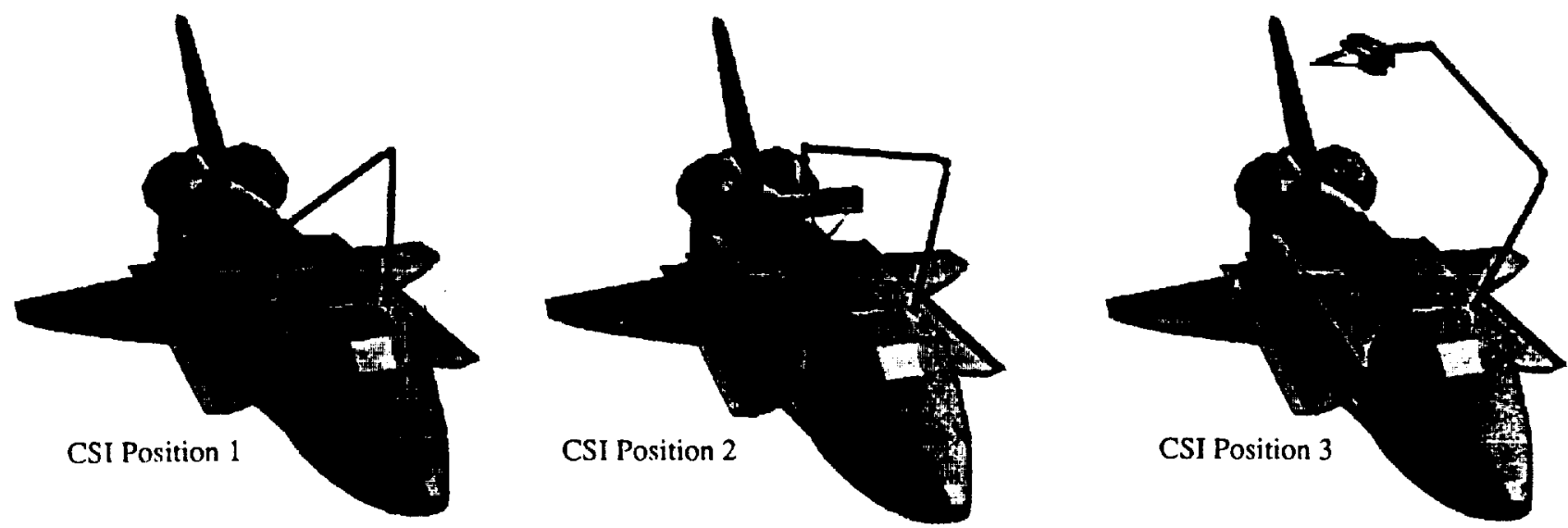

Figure 3 - RMS study configurations.

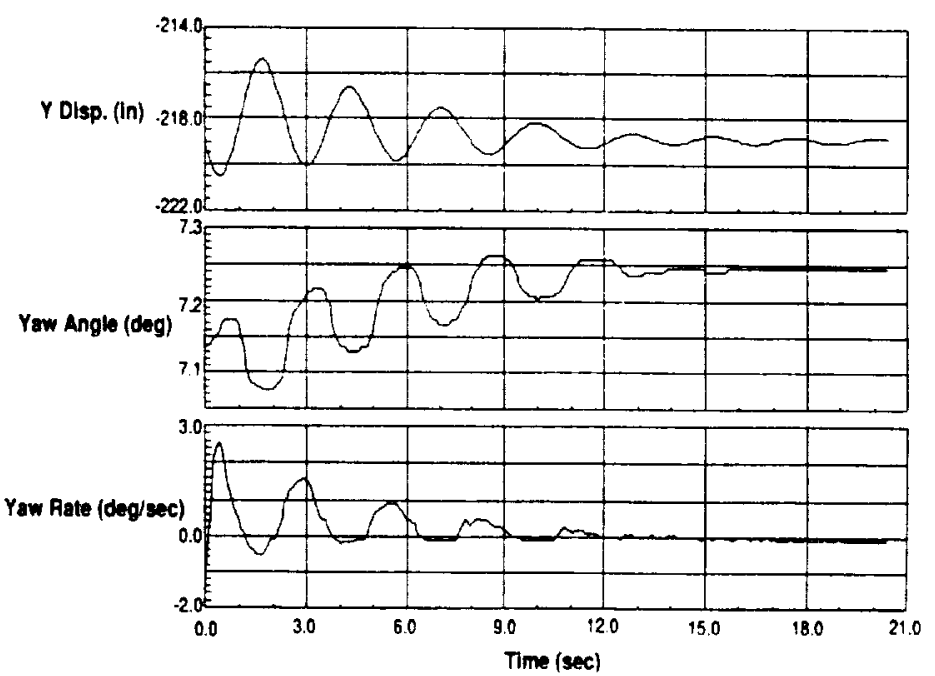

Figure 4 - Typical RMS Response and Sensor Outputs. 


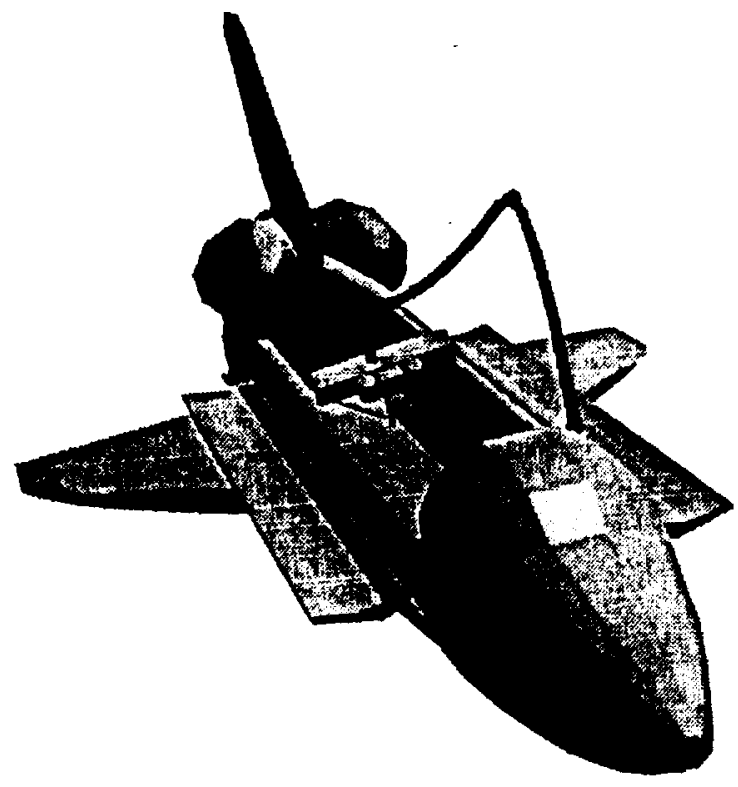

Figure 5 - RMS structural mode shape.

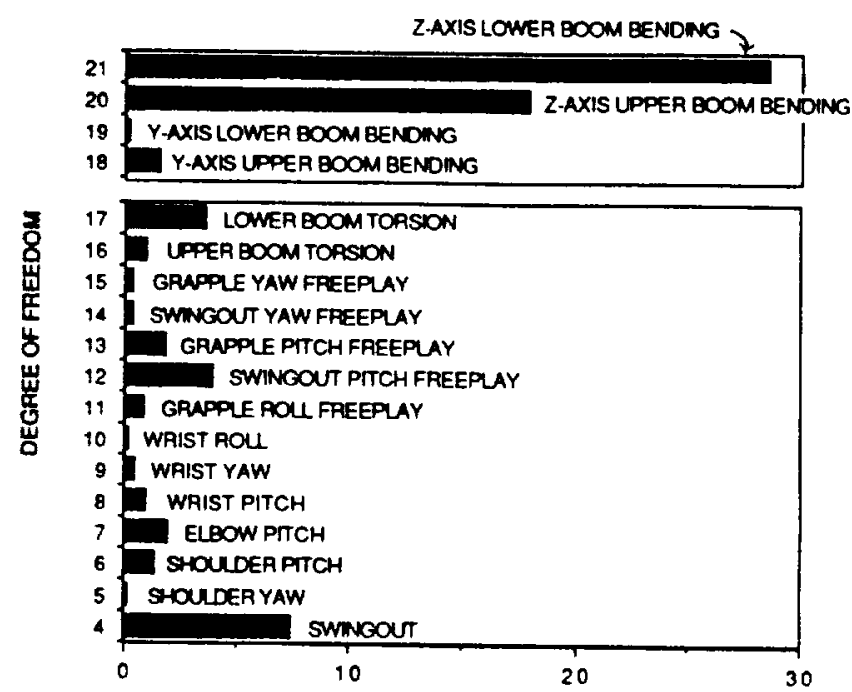

Figure 6 - RMS structural mode contributions.

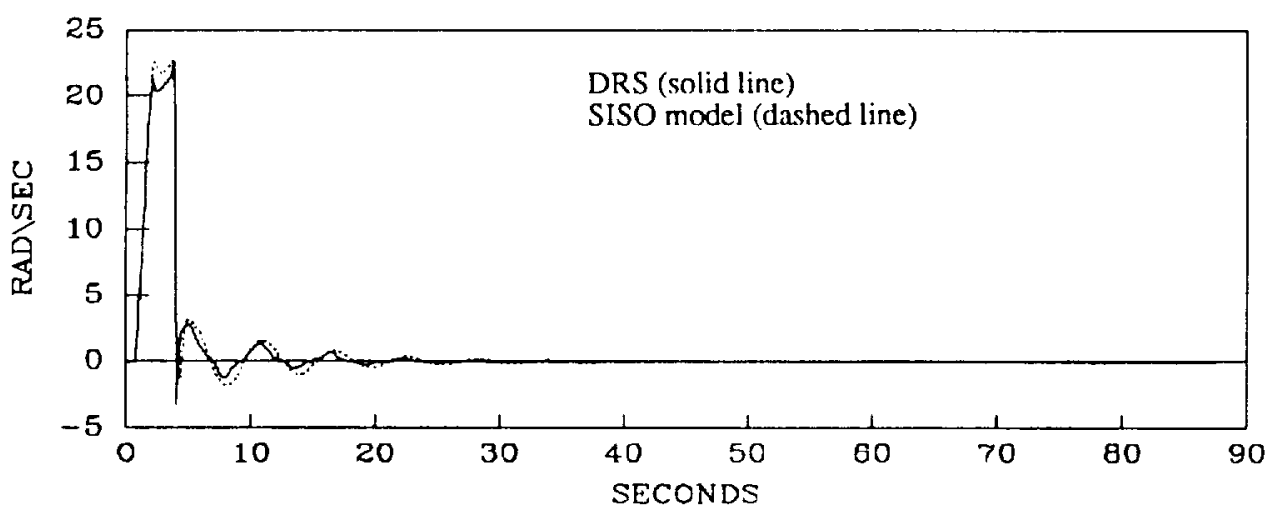

Figure 7 . The SISO System Identification results for the shoulder-yaw tachometer.

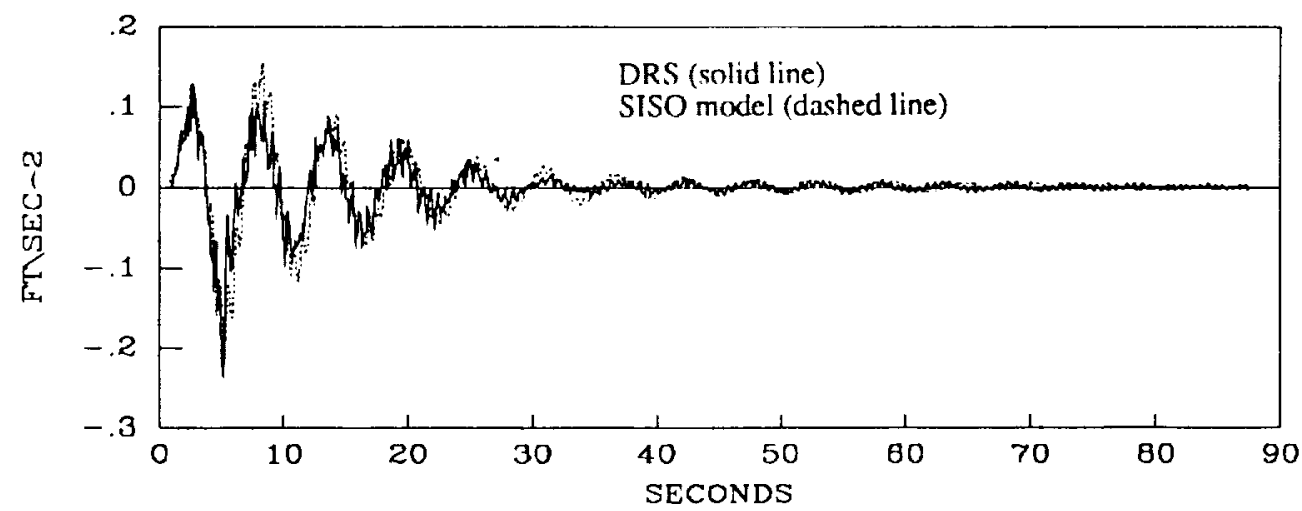

Figure 8 - The SISO System Identification results for the tip accelerometer (y axis). 


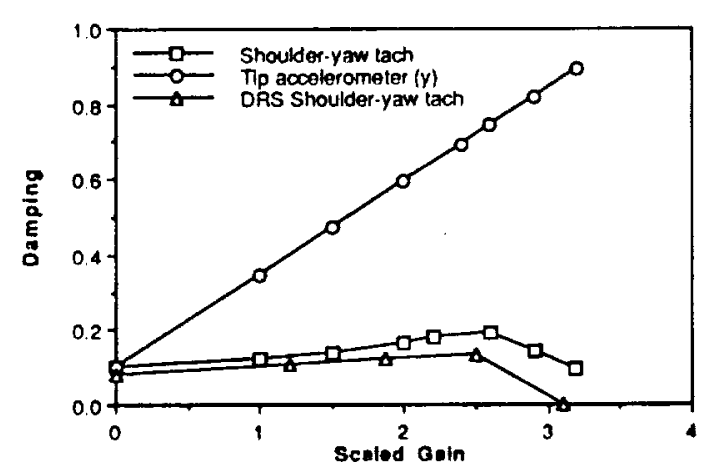

Figure 9 - Damping as a function of scaled gain for CSI position 1 using the shoulder-yaw joint.

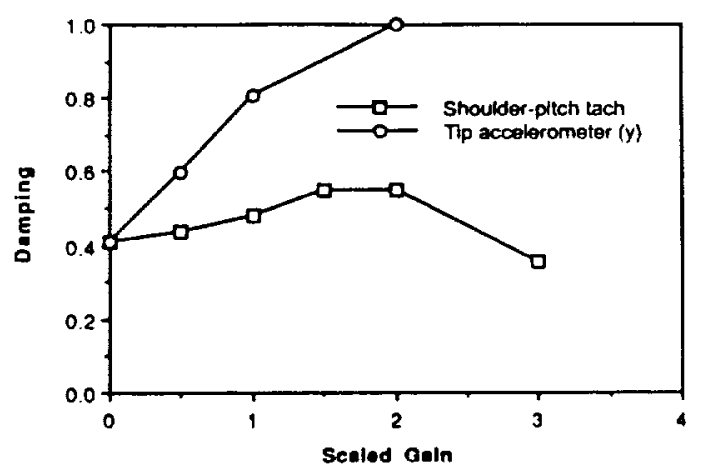

Figure 10 . Damping as a function of scaled gain for CSI position 1 using the shoulder-pitch joint.

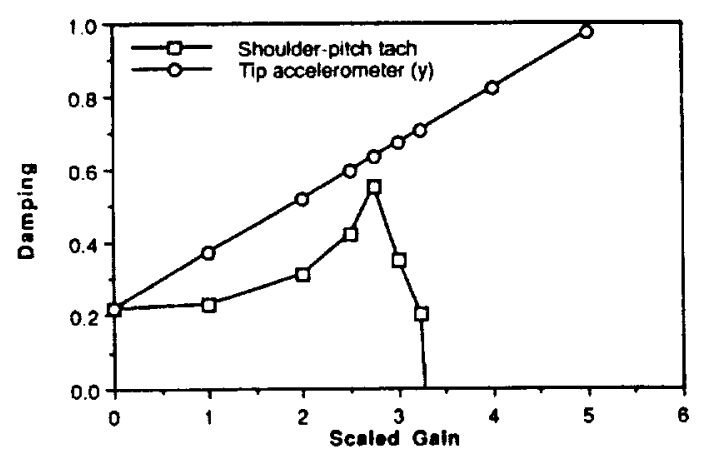

Figure 11 - Damping as a function of scaled gain for CSI position 3 using the shoulder-pitch joint.

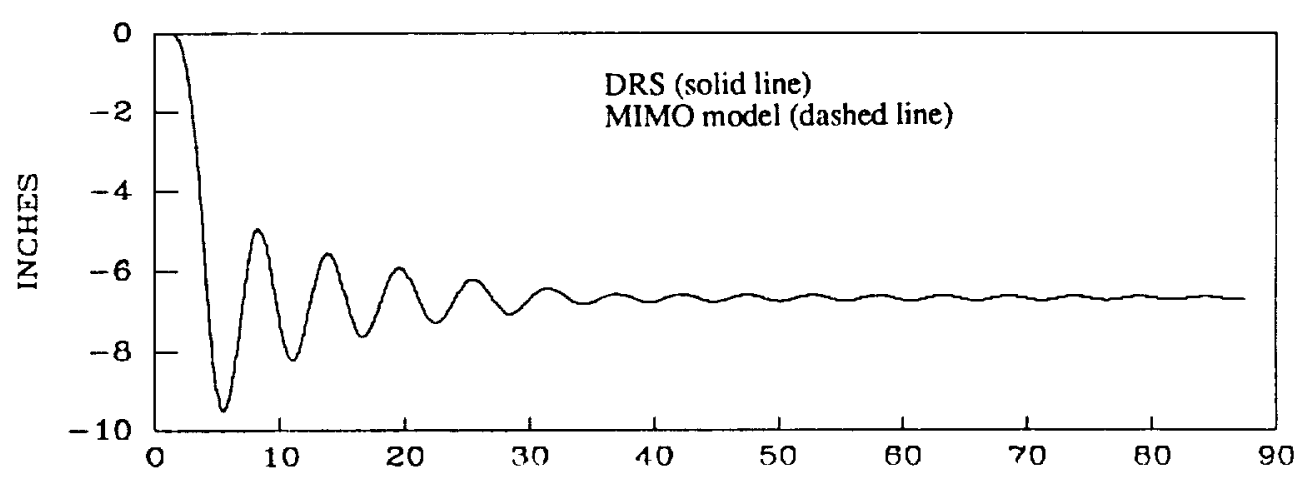

Figure 12 . The MIMO system identification results for the tip position (y axis).

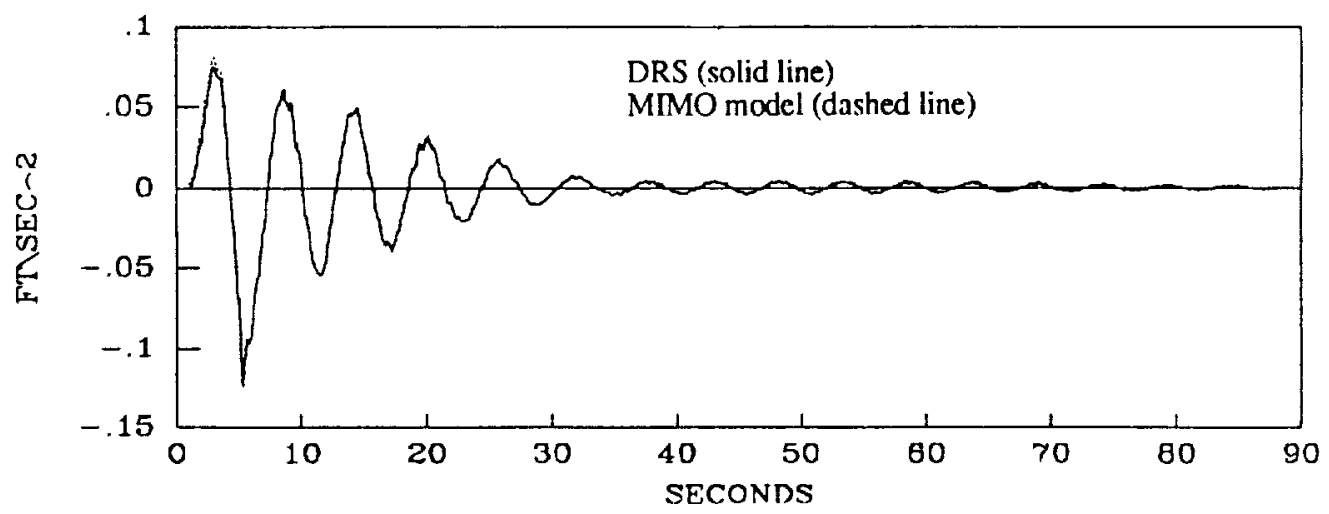

Figure 13 - The MIMO system identification results for the tip accelerometer (y axis). 


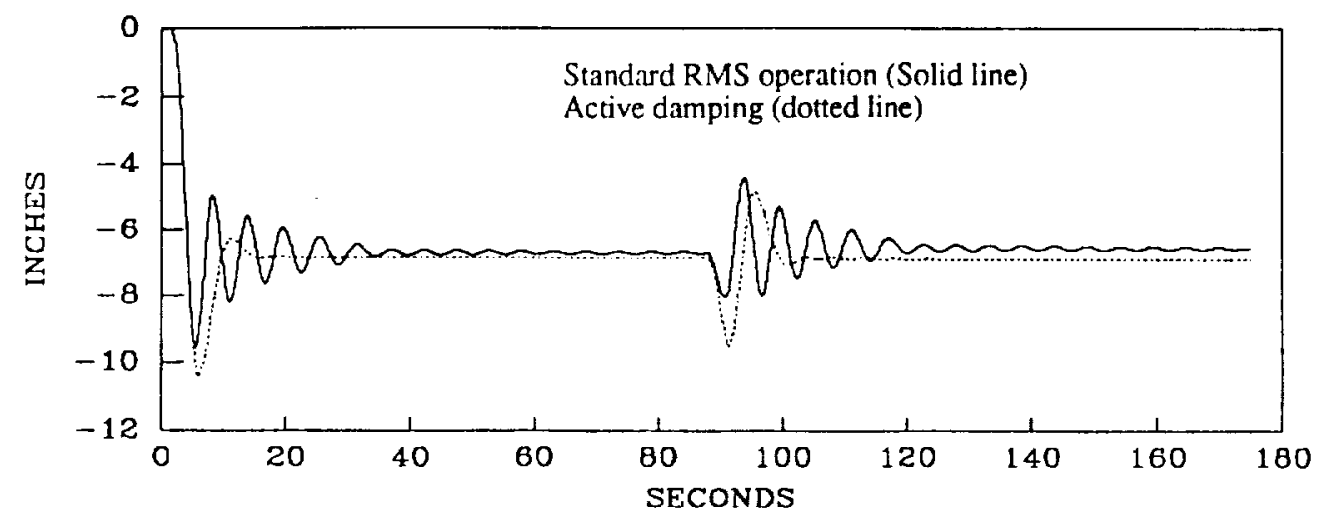

Figure 14 . Tip position following 3 second pulse command. After 90 seconds the shuttle thrusters are fired for 6 seconds.

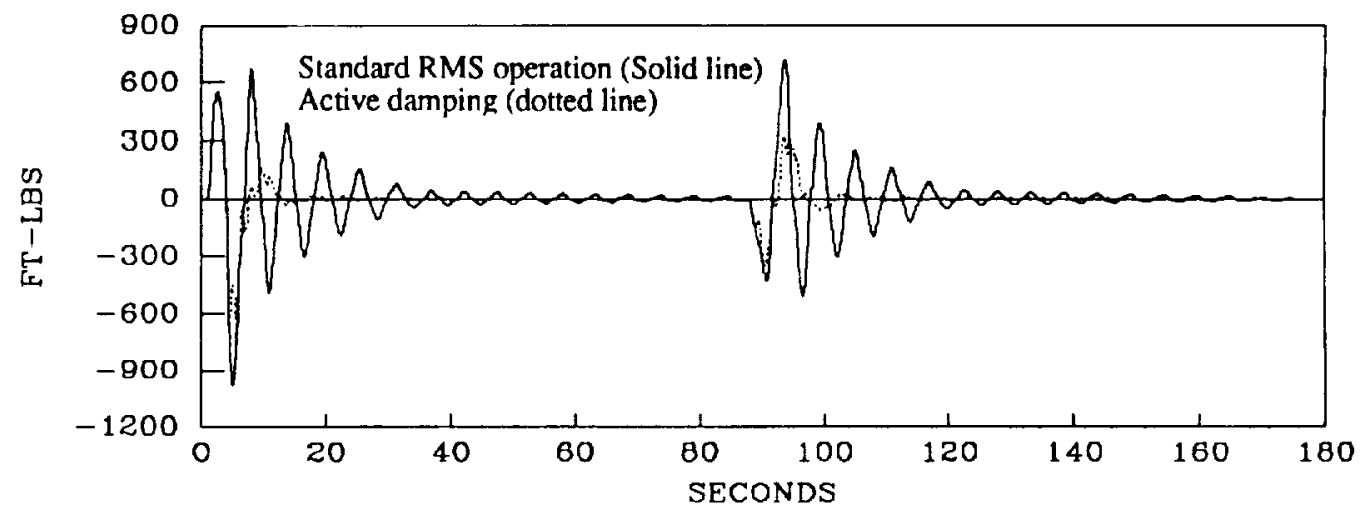

Figure 15 - Shoulder Yaw servo torque following 3 second pulse command. After 90 seconds the shuttle thrusters are fired for 6 seconds.

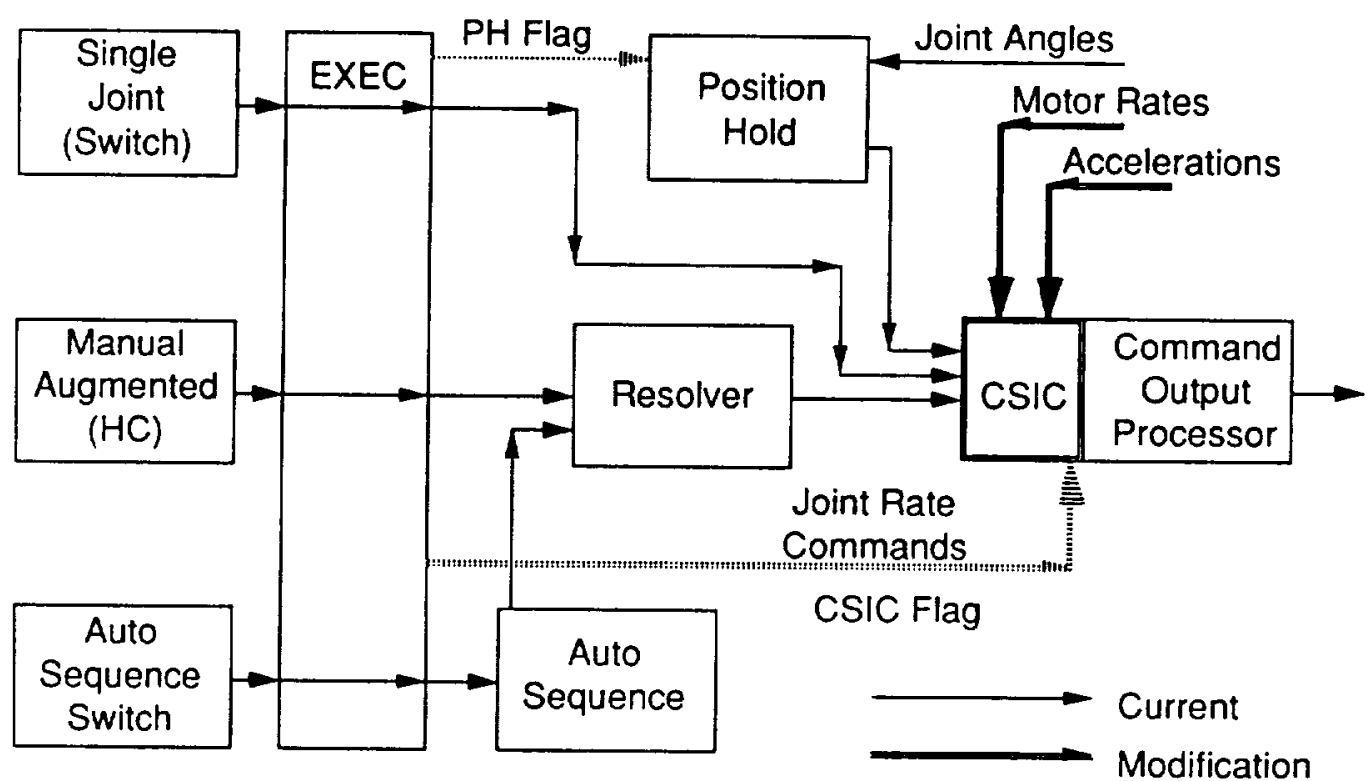

Figure 16 - Proposed CSI controller implementation in Shuttle GPC Software. 


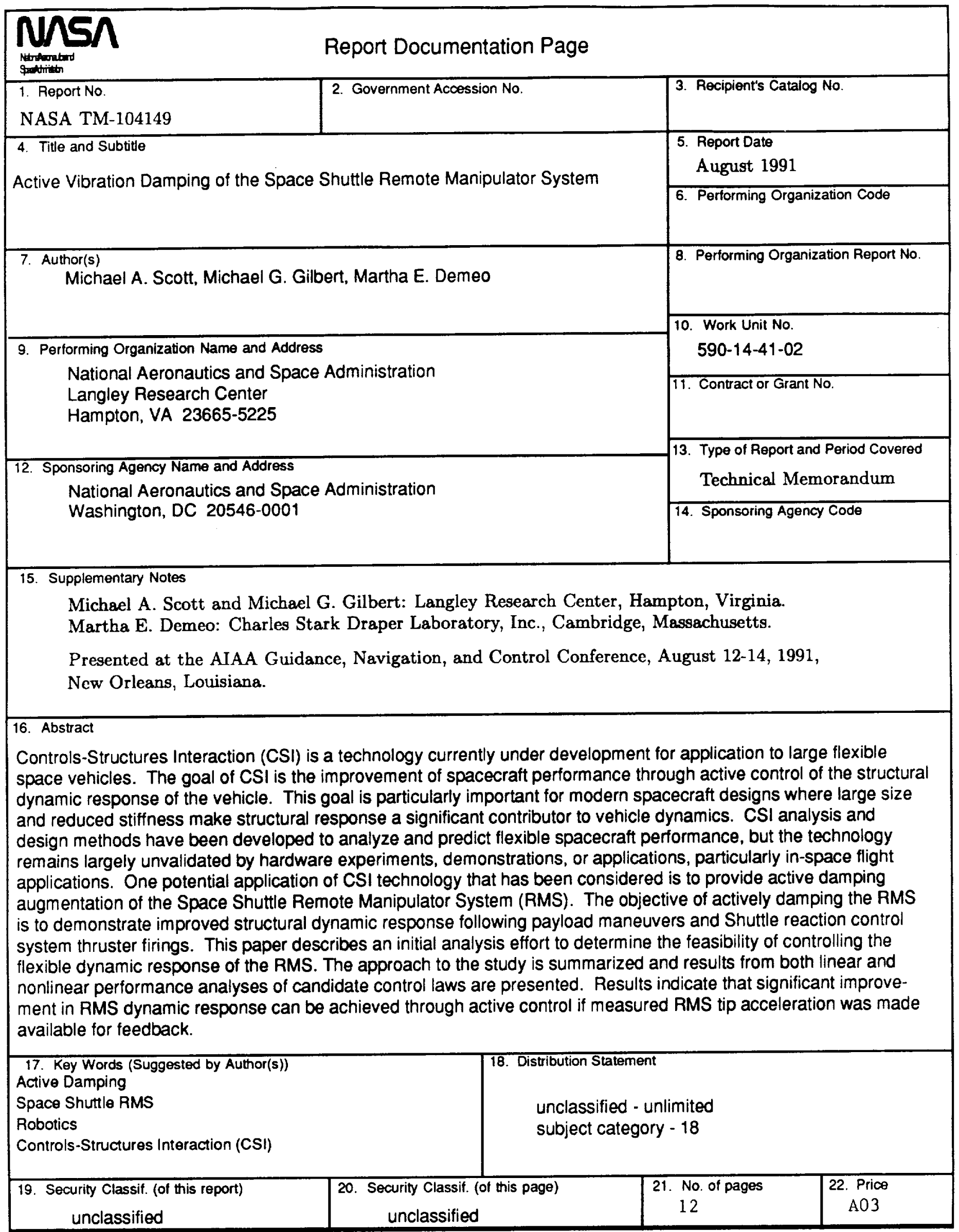

NASA FORM 1626 OCT 86 For sale by the National Technical information Service, Springfield, VA 22161-2171 